\title{
ANÁLISES MELISSOPALINOLÓGICAS, FÍSICO-QUÍMICAS, ATIVIDADE ANTIRRADICALAR E PERFIL QUÍMICO POR UPLC-DAD-QTOF-MS/MS DOS MÉIS DE Frieseomelitta doederleini (ABELHA BRANCA): COMPARAÇÃO COM OS FENÓLICOS PRESENTES NAS FLORES DE Mimosa tenuiflora (JUREMA PRETA)
}

\author{
Rogelio M. Santistebana, Sonia P. Cabrera ${ }^{a}$, José F. Neto ${ }^{b}$, Eva M. S. Silva ${ }^{b}$, Rebert C. Correia ${ }^{c}$, Rodolfo F. Alves ${ }^{\text {, Francisco }}$ \\ de A. R. dos Santos ${ }^{\text {, Celso A. Camara }}{ }^{a}$ e Tania M. S. Silva ${ }^{a, *,(1)}$ \\ aDepartamento de Química, Universidade Federal Rural de Pernambuco, 52171-900 Recife - PE, Brasil \\ ${ }^{b}$ Colegiado de Zootecnia, Universidade Federal do Vale do São Francisco, 56300-990 Petrolina - PE, Brasil \\ ${ }^{\mathrm{c}}$ Embrapa Semiárido, 56302-970 Petrolina - PE, Brasil \\ ${ }^{d}$ Departamento de Ciencias Biológicas, Universidade Estadual de Feira de Santana, 44036-900 Feira de Santana - BA, Brasil
}

Recebido em 06/06/2019; aceito em 24/07/2019; publicado na web em 30/08/2019

\begin{abstract}
MELISSOPALINOLOGICAL, PHYSICOCHEMICAL ANALYSIS, ANTIRADICALAR ACTIVITY AND CHEMICAL PROFILE BY UPLC-DAD-qTOF-MS/MS OF FRIESEOMELITTA DOEDERLEINI (ABELHA BRANCA) HONEY BEE: COMPARISON WITH THE FLOWERS OF MIMOSA TENUIFLORA (JUREMA PRETA). Melissopalynological, physicochemical analysis, mineral content, antiradical activity and profile of compounds by Ultra-Performance Liquid Chromatography coupled with a Diode Array Detector and quadrupole Time of Flight Mass Spectrometry (UPLC-DAD-qTOF-MS/MS) of Frieseomelitta doederleini honeys from semiarid region of the Northeast of Brazil are presented. The melissopalynological analysis showed two principal pollen types of Mimosa tenuiflora (jurema preta) and Shinus sp (aroeira). The chemical profile data obtained by UPLC-DAD-qTOF-MS/MS of the Mimosa tenuiflora flowers (principal pollen of honey) and the five samples of $F$. doederleini honeys both flowers and honey showed similar profile. Abelha branca honeys samples had similar characteristic profile of phenolic compounds (flavonoids), and also antiradical activity. The main flavonoids identified in the honey samples in the jurema preta flowers were flavonols/flavone and flavanols/flavanones aglycones. All the flavonoids identified showing that the nectar collected by the bees may be from this plant species. The antiradical activity of the honey samples strongly correlated with their phenolic content. Among the minerals analyzed, potassium was the most abundant, followed by magnesium.
\end{abstract}

Keywords: stingless bee; honey; Mimosa tenuiflora; chemical profile; antiradical activity.

\section{INTRODUÇÃO}

Os meliponíneos também conhecidos como 'abelhas indígenas sem ferrão’ (família Apidae, subtribo Meliponina) compõem-se de várias centenas de espécies em todas as regiões tropicais do mundo, bem como nas regiões subtropicais do hemisfério sul. São abelhas de minúsculas a médias, em geral robustas. Todas as espécies são eussociais e os ninhos são, em geral, construídos em cavidades pré-existentes (ocos de árvores, ninhos abandonados de cupins e formigas, etc.), mas algumas espécies constroem ninhos expostos. Os meliponíneos possuem o ferrrão atrofiado, são incapazes de ferroar e são conhecidas também como abelhas nativas. ${ }^{1}$

No Brasil existem mais de 300 espécies de abelhas nativas encontradas principalmente nas regiões Norte e Nordeste. Na caatinga brasileira são conhecidas 187 espécies, sendo que a maioria é considerada como rara. ${ }^{2}$ As abelhas sociais nativas sem ferrão como jandaíra, jati, amarela, moça-branca, irapuá, cupira, mandaçaia, remela, canudo, limão, munduri e a introduzida Apis mellifera, também conhecida como abelha de mel, abelha europa e abelha africanizada, são as mais abundantes. Outras espécies de abelhas de hábitos solitários também são abundantes e de grande importância ecológica. ${ }^{3}$

As abelhas sem ferrão do gênero Frieseomelitta (Meliponinae, Trigonini), são exclusivamente neotropicais com ampla distribuição geográfica, ocorrendo desde o Sudeste do México até a região Sudeste do Brasil. As espécies podem ser encontradas em florestas, cerrado, caatinga e regiões montanhosas atingindo até $1600 \mathrm{~m}$ de altitude no México. ${ }^{4}$

\footnotetext{
*e-mail: sarmentosilva@gmail.com
}

A abelha sem ferrão Frieseomelitta doederleini, conhecida como abelha branca, asa branca e mané-de-abreu é endêmica do bioma caatinga na região Nordeste do Brasil, podendo ser encontrada nos estados da Bahia, Ceará, Maranhão, Paraíba, Pernambuco, Piauí e Rio Grande do Norte. A F. Doederleini é uma abelha de pequeno porte com o ninho em forma de cacho de uva e produz em torno de 2 litros de mel por colônia/ano a partir de espécies vegetais nativas da região seca. Com poucos dados na literatura, as informações sobre a espécie no bioma caatinga são repassadas pelas observações da população local. O mel de abelha branca é muito valorizado pela população, principalmente no estado da Bahia.

Os méis das abelhas nativas sem ferrão presentes no território brasileiro são produtos únicos e peculiares da biodiversidade do País, muito valorizados pela cultura popular. Apresentam características próprias como maior teor de umidade, geralmente são mais ácidos e menos viscosos que o mel proveniente da espécie exótica Apis mellifera. Estas diferenças levam a necessidade de uma norma específica para o estabelecimento de parâmetros físico-químicos que sejam referência tanto para controle de qualidade como para sua comercialização. ${ }^{5}$ Devido ao endemismo de determinadas espécies de abelhas nativas em regiões diferentes do País, alguns estados têm publicado resoluções com as normas técnicas para o mel de abelhas nativas específicas como, por exemplo, a norma técnica para o padrão de qualidade do mel de abelhas nativas do estado de São Paulo. ${ }^{6}$ Por essa razão existe a necessidade da realização dos testes físico-químicos para méis de outras espécies de abelhas nativas, especialmente para abelha branca. A necessidade de regulamentação técnica para beneficiamento e controle de qualidade da produção do mel das abelhas nativas presentes no Nordeste do Brasil pode promover uma cascata de benefícios 
como geração de emprego e renda, fixação do homem no campo, incremento nas economias locais e regionais, valorização da biodiversidade, conservação dos ecossistemas e incremento e promoção de uma agricultura mais sustentável.

A espécie vegetal Mimosa tenuiflora, conhecida popularmente por jurema-preta, é uma árvore conhecida na caatinga pela presença de espinhos em seus ramos e floresce durante um longo período do ano, porém predominantemente durante a estação seca. Suas inflorescências são reunidas em espigas, formadas por flores brancas, pequenas e suavemente perfumadas, que fornecem recursos florais, pólen e néctar, para muitas espécies de abelhas nativas (incluindo a abelha branca), vespas, moscas e outros insetos, sendo muito importante para a manutenção da biodiversidade e funcionamento do ecossistema. Além disso, devido ao seu crescimento rápido e a sua capacidade de rebrota essa espécie é muito importante para a restauração de áreas degradadas. ${ }^{3}$

Este trabalho está inserido no projeto MeliApis: Projeto de inserção e capacitação da mulher em atividade da agricultura familiar em municípios do território do sertão próximo ao Rio São Francisco, sendo a continuação dos estudos com os produtos apícolas e meliponícolas. ${ }^{7-13}$ Neste estudo, com as amostras dos méis de abelha branca foram realizadas análises físico-químicas, químicas por Cromatografia a Líquido de Ultra-eficiência acoplada com detectores de Arranjo de Diodo e Espectrômetro de Massas tipo triploquadrupolo e Tempo de Vôo (UPLC-DAD-qTOF-MS/MS), teor de minerais e atividade antirradicalar, como também foi realizada a análise e comparação do perfil químico das flores de Mimosa tenuiflora (jurema preta), principal espécie vegetal que apresentou o tipo polínico predominante nos méis de abelha branca.

\section{PARTE EXPERIMENTAL}

\section{Análises físico-químicas}

Para os testes físico-químicos utilizou-se balança analítica (AW220, Shimadzu, Kyoto, Japan), Refratômetro (RHB-90ATC, MegaBrix, Paraná), Estufa (TE393/2-MP Tecnal, São Paulo, Brasil), Microondas (Mars Xpress, CEM, Matthews, NC, EUA), Phmetro e Condutivimetro (AZ 86505, Az, China), Espectrofotômetro UV-Visivel (Lambda 45, Perkim Elmer, São Paulo, Brasil), mufla (1615, Fornitec) e equipamento medidor da atividade de água (AquaLab 4TE, Decagon Devices, WA, USA). Os solventes e reagentes utilizados para estas analises foram: hidróxido de sodio (Dinâmica, São Paulo, Brasil), ferrocianeto de potássio, azul de metileno, bisssulfito de sodio (Dinâmica, São Paulo, Brasil), solução tampão pH 4, 7 e 9 (Haloquímica LTDA, São Paulo, Brasil), solução de Fehling A e Fehling B (Teclab, São Paulo, Brasil), solução de Carrez I e II (Dinâmica, São Paulo, Brasil) e ácido nítrico (Merck, Dramstadt, Alemanha). As amostras dos méis foram submetidas as

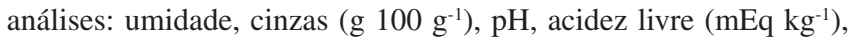
condutividade elétrica $\left(\mu \mathrm{S} \mathrm{cm}^{-1}\right)$, hidroximetilfurfural ( $\mathrm{HMF}, \mathrm{mg} \mathrm{kg}^{-1}$ ),

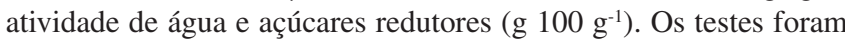
realizados em triplicata de acordo com os métodos adotado pelo Instituto Adolfo Lutz. ${ }^{14}$

\section{Coleta das amostras e análises melissopalinológicas}

Cinco amostras de mel de abelha branca (1-5) foram coletadas no município de Remanso-Bahia, Nordeste do Brasil em dezembro de 2015. As amostras foram armazenadas sob refrigeração à temperatura de $5{ }^{\circ} \mathrm{C}$ até o início das análises. As análises melissopalinológicas foram realizadas de acordo com Erdtman (1960). ${ }^{15}$ Os tipos polínicos foram identificados por comparação com lâminas depositadas na palinoteca do Laboratório de Micromorfologia Vegetal, Departamento de Ciências Biológicas da Universidade Estadual de Feira de Santana e com o auxílio dos catálogos polínicos disponíveis. Os tipos polínicos foram classificados como pólen dominante $(>45 \%)$, pólen acessório $(\leq 45 \% \geq 15 \%)$, pólen isolado importante $(\leq 15 \% \geq 3 \%)$ e pólen isolado ocasional (<3\%). As flores de Mimosa tenuiflora (jurema preta) foram coletadas no sítio Riacho, município de Vieirópolis, estado da Paraíba. Uma exsicata está depositada no Herbário da Universidade Federal Rural de Pernambuco, número 53530.

\section{Determinação do teor de minerais}

As amostras dos méis foram digeridas em digestor de amostras por microondas com controle de temperatura. A $500 \mathrm{mg}$ da amostra foram adicionados $5 \mathrm{~mL}$ de ácido nítrico concentrado, utilizando-se o seguinte método: $800 \mathrm{~W} \mathrm{~min}{ }^{-1}$ por 5 minutos com temperatura limitada a $120{ }^{\circ} \mathrm{C}, 5$ minutos a $800 \mathrm{~W}$ com temperatura limitada a $160{ }^{\circ} \mathrm{C}$ e resfriamento por 20 minutos. A solução foi diluída com $15 \mathrm{~mL}$ de água desionizada antes da análise por absorção atômica utilizando lâmpadas de catodo oco individuais e padrões a $100 \mu \mathrm{g} \mathrm{mL} \mathrm{m}^{-1} \mathrm{ppm}$ dos minerais certificados (Qhemis-Hexis, São Paulo, Brasil). A análise elementar $(\mathrm{Cu}, \mathrm{Fe}, \mathrm{K}, \mathrm{Mn}, \mathrm{Zn}, \mathrm{Cr}, \mathrm{Na}, \mathrm{Mg}, \mathrm{Cd}, \mathrm{Pb}$ e $\mathrm{Ca})$ foi realizada em espectrofotômetro Varian AA 240 (Victoria, Austrália) por espectrometria de absorção atômica com chama (F ASS). As curvas de calibração com cinco concentrações para os metais foram construídas e todas as análises em triplicata. Todos os ajustes instrumentais foram seguidos de acordo com o manual do fabricante. Os valores obtidos para o conteúdo de metais nas amostras foram calculados como $\mathrm{mg} \mathrm{kg}^{-1}$ de mel.

Extração dos compostos orgânicos do mel em fase sólida (SPE) e das flores de Mimosa tenuiflora

Uma alíquota de $10 \mathrm{~g}$ do mel foi solubilizada sob agitação em $2 \mathrm{~mL}$ de água acidificada ( $\mathrm{pH}=2 \mathrm{com} \mathrm{HCl}$ ). Os compostos foram extraídos usando-se cartucho de extração em fase sólida SPE C-18, ${ }^{16}$ que foram previamente ativados utilizando-se $6 \mathrm{~mL}$ de metanol e $6 \mathrm{~mL}$ de água ultra pura. Após este procedimento, a amostra do mel foi submetida a extração com $10 \mathrm{~mL}$ de água ultra pura para remoção dos açúcares e outros constituintes polares do mel. A fração dos compostos retidas no cartucho foi eluída com $10 \mathrm{~mL}$ de metanol e em seguida, o solvente foi evaporado obtendo-se as frações com pesos que variaram de 10,97-12,92 mg.

As flores de Mimosa tenuiflora (89,0 g) foram extraídas com etanol e a solução foi evaporada em rotavapor. Parte do extrato etanolólico ( $3,1 \mathrm{~g})$ foi submetido a partição com $\mathrm{MeOH}: \mathrm{H}_{2} \mathrm{O}(1: 1)$, hexano e acetato de etila. A fração acetato de etila $(1,0 \mathrm{~g})$ obtida após evaporação do solvente foi submetida a análise por UPLC-DAD-qTOF-MS/MS para comparação do perfil químico com as amostras dos méis.

\section{Determinação do teor de fenólicos, flavonóides totais e atividade antirradicalar}

O teor de fenólicos totais foi determinado com o reagente de Folin-Ciocalteu com modificações ${ }^{11}$ utilizando o ácido gálico como composto fenólico padrão. Uma alíquota de $100 \mu \mathrm{L}$ das soluções de mel diluído em água (100 $\left.\mathrm{mg} \mathrm{mL}^{-1}\right)$ foram transferidos para um Eppendorff, adicionados $10 \mu \mathrm{L}$ do reagente de Folin-Ciocalteau e 360 $\mu \mathrm{L}$ de água destilada, agitando-se por $1 \mathrm{~min}$. Em seguida, $30 \mu \mathrm{L}$ de $\mathrm{Na}_{2} \mathrm{CO}_{3}(15 \%)$ foram acrescentados à mistura e agitados por 30 segundos, resultando na concentração final de $20 \mathrm{mg} \mathrm{mL}^{-1}$. Após duas horas, a absorbância de cada amostra foi medida em espectrofotômetro de Elisa UV-Vis em 760 nm, empregando-se placas de 96 poços. 
As análises foram realizadas em triplicata e o teor de fenólicos totais foi determinado por interpolação da absorbância das amostras contra uma curva de calibração construída com soluções do padrão do ácido gálico, o resultado foi expresso em miligrama equivalente de ácido gálico por grama de mel (mg EAG g $\left.{ }^{-1}\right)$

O teor de flavonoides totais foi determinado usando o método de Vermerris e Nicholson. ${ }^{17} 250 \mu \mathrm{L}$ das soluções de mel diluído em água (100 $\mathrm{mg} \mathrm{mL}^{-1}$ ) foi transferido para um Eppendorff, adicionando-se, em seguida, $100 \mu \mathrm{L}$ de cloreto de alumínio $\left(\mathrm{AlCl}_{3} 5 \%\right)$ e completando-se o volume para $500 \mu \mathrm{L}$ com água destilada. Após 30 minutos, a absorbância de cada amostra foi medida em espectrofotômetro de Elisa UV-Vis em $425 \mathrm{~nm}$, empregando-se placas de 96 poços. As análises foram realizadas em triplicata e o teor de flavonoides totais foi determinado por interpolação da absorbância das amostras contra uma curva de calibração construída com soluções do padrão de quercetina e expressos em miligrama equivalente de quercetina por grama de mel (mg EQ g $\left.{ }^{-1}\right)$.

A atividade antirradicalar foi determinada utilizando os radicais DPPH e ABTS. O teste com o DPPH foi realizado de acordo com a metodologia descrita por Silva et al. ${ }^{9}$ com pequenas modificações. As amostras dos méis $\left(1,0 \mathrm{mg} \mathrm{mL}^{-1}\right)$ foram preparadas pela adição de quantidades apropriadas a $450,0 \mu \mathrm{L}$ da solução de DPPH (23,6 $\mu \mathrm{g} \mathrm{mL}^{-1}$ em EtOH) e o volume foi completado para $500,0 \mu \mathrm{L}$ com EtOH, obtento-se concentrações finais que variaram de 1,0 a $400,0 \mu \mathrm{g} \mathrm{mL}^{-1}$. Após 30 minutos de agitação em aparelho de ultrassom ao abrigo da luz, as amostras foram submetidas as análises em espectrofotômetro Elisa UV-Vis a $517 \mathrm{~nm}$. A percentagem da atividade sequestradora foi calculada pela equação: \% $\mathrm{AS}=100 \mathrm{x} \Delta \mathrm{hx} / \mathrm{hc}$, $\mathrm{hc}=$ absorbância controle, $\mathrm{hx}=$ absorbância teste, $\Delta \mathrm{hx}=\mathrm{hc}-\mathrm{hx} . \mathrm{O}$ ácido ascórbico (concentrações variando entre 0,5 a $4,0 \mu \mathrm{g} \mathrm{mL}^{-1}$ ) foi utilizado como controle positivo.

O ensaio da atividade sequestradora do cátion radical ABTS foi realizado seguindo a metodologia de Silva et al., ${ }^{13}$ com pequenas modificações. $\mathrm{O}$ cátion radical $\mathrm{ABTS}^{*+}$ foi preparado pela reação com $7 \mathrm{mmol} \mathrm{L}^{-1}$ de ABTS $(2,5 \mathrm{~mL})$ e persulfato de potássio $140 \mathrm{mmol} \mathrm{L}^{-1}$ $(44 \mu \mathrm{L})$. A mistura ficou ao abrigo da luz em temperatura ambiente durante 14 horas. Para o ensaio, $1 \mathrm{~mL}$ da solução do radical ABTS foi diluída em EtOH até obter uma absorbância $\pm 0,7$ a $734 \mathrm{~nm}$. Foram adicionadas quantidades apropriadas das amostras a $450,0 \mu \mathrm{L}$ da solução do radical ABTS e o volume completado para 500,0 $\mu \mathrm{L}$ com EtOH, obtendo-se concentrações que variaram de 1,0 a 100,0 $\mu \mathrm{g} \mathrm{mL} \mathrm{mL}^{-1}$. Após 6 minutos de agitação em aparelho de ultrassom ao abrigo da luz, as amostras foram submetidas as análises por espectrofotômetro Elisa UV-Vis no comprimento de onda de $734 \mathrm{~nm}$. A percentagem da atividade sequestradora foi calculada pela equação: \% AS = $100 \times \Delta \mathrm{hx} / \mathrm{hc}, \mathrm{hc}=$ absorbância controle, $\mathrm{hx}=$ absorbância teste, $\Delta \mathrm{hx}=\mathrm{hc}-\mathrm{hx}$. Como controle positivo foi utilizado o Trolox $\left(1,0-6,0 \mathrm{mg} \mathrm{mL}^{-1}\right)$.

\section{Análises por UPLC-DAD-qTOF-MS/MS}

Os experimentos analíticos de LC-MS foram realizados utilizando-se um cromatógrafo líquido de ultra eficiência ACQUITY UPLC H-Class (Waters Corporation, Milford, MA, EUA), acoplado a um espectrômetro de massas Quadupolo-Tof (Xevo G2-XS QTof, Waters, EUA) com ionização por eletrospray (ESI). As separações cromatográficas foram realizadas utilizando-se uma coluna ACQUITY UPLC $^{\text {TM }}$ BEH C18 (2,1 x 50 mm, $1,7 \mu \mathrm{m}$, Waters, EUA) a $40{ }^{\circ} \mathrm{C}$. A fase móvel binária consistiu de uma solução aquosa de ácido fórmico (fase móvel A) e acetonitrila com $0,1 \%$ de ácido fórmico (fase móvel B). O gradiente de eluição utilizado foi: 0.0-8.0 min, 10-50\% de B; 8,0-9,0 mim, 50\%-95\% de B, com o fluxo de $0,4 \mathrm{~mL} \mathrm{~min}^{-1}$ e volume de injeção de 5,0 $\mu \mathrm{L}$. O monitoramento das análises foi realizado nos comprimentos de 290 e 320 nm (UPLC-DAD). O espectrômetro de massas foi operado em modo negativo de ionização (ESI-), sendo a detecção realizada no método centróide $\mathrm{MS}^{\mathrm{E}}$ em faixa de variação 50-1200 Da. A voltagem do capilar foi ajustada em $3,0 \mathrm{kV}$ para ESI, gás de dessolvatação $\left(\mathrm{N}_{2}\right) 800 \mathrm{~L} \mathrm{~h}^{-1}$ e $600{ }^{\circ} \mathrm{C}$, taxa do fluxo de gás do cone $100 \mathrm{~L} \mathrm{~h}^{-1}$, temperatura da fonte $120{ }^{\circ} \mathrm{C}$, energia de colisão em rampa de 10-30 eV. Todas as análises foram realizadas utilizando o lockspray para garantir a precisão e reprodutibilidade dos valores de massas. Leucina-encefalina $\left(200,0 \mathrm{pg} \mathrm{mL}^{-1}\right)$ foi utilizado como padrão/referência para calibração. A aquisição e análise dos dados foram realizadas utilizando-se o software Waters MassLynx. Luteolina, quercetina, narigenina, apigenina e ácido elágico foram obtidos da Sigma-Aldrich (Hamburg, Alemanha), 7-metoxi-narigenina ${ }^{13}$ e isorametina ${ }^{9}$ foram previamente isolados e identificados.

\section{Análises estatísticas}

Os resultados dos testes foram obtidos em triplicata e expressos como média \pm desvio padrão da média. Foi utilizado o programa GraphPad Prism 7.0. (GraphPad ® Software DEMO, San Diego, Califórnia, EUA)

\section{RESULTADOS E DISCUSSÃO}

\section{Análises melissopalinológicas}

O grão de pólen apresenta parede externa quimicamente estável e morfologicamente variada, o que permite, através da análise palinológica das cargas de pólen coletadas pelas abelhas, a identificação dos diversos tipos polínicos constituintes, verificação dos períodos de produção e a determinação da origem botânica e geográfica das amostras. ${ }^{18}$

A análise palinológica das cinco amostras de méis analisadas (Tabela 1) mostrou a predominância de dois principais tipos polínicos, um relativo a plantas do gênero Schinus sp (Anacardeaceae), com variação de 11 a 36\%, e outro de Mimosa tenuiflora (Fabaceae), com variação de 29 a $34 \%$, sendo classificado, portanto, como heterofloral (Tabela 1). O pólen apícola ou meliponícola só é considerado monofloral quando apresenta predominância com mais de $45 \%$ de uma determinada espécie vegetal.

Estudo realizado por Teixeira et al. ${ }^{19}$ mostrou que espécies de Frieseomelitta sp apresentam padrão de forrageio semelhante ao observado para outros grupos de abelhas eussociais, visitando diversas espécies vegetais, mas concentrando a visita em poucas espécies. Foi observado que essas abelhas visitam espécies da família Caesalpiniaceae em áreas da caatinga nos estados da Bahia e Paraíba. Espécies vegetais pertencentes a esta família são importantes fontes de néctar e pólen. Da mesma forma, espécies vegetais das famílias Malpighiaceae e Anacardiaceae abundantes na região são bastante visitadas pelas abelhas $F$. doederleini. ${ }^{19}$ Essas observações podem ser vistas na Tabela 1 , em que se verifica que espécies vegetais destas famílias foram visitadas pela abelha branca.

\section{Análises físico-químicas}

As amostras dos méis de abelha branca mostrou aspecto viscoso, coloração marrom escura, sabor de "mel melaço de cana de açúcar" e aromático. O resultado das análises físico-químicas do mel de abelha branca estão apresentados na Tabela 2. Os valores de $\mathrm{pH}$ variaram de 3,68 a 3,92 quando comparado com os valores reportados para os méis de abelhas nativas do estado de São Paulo. ${ }^{6}$ Os valores de $\mathrm{pH}$ baixo dos méis inibe a presença e crescimento de micro-organismos. Esse parâmetro é muito importante para o armazenamento e influencia na 
Tabela 1. Análises melissopalinológicas das amostras do mel de abelha branca

\begin{tabular}{|c|c|c|c|c|c|}
\hline \multirow{2}{*}{ Tipos polínicos } & \multicolumn{5}{|c|}{ Méis de abelha branca (\%) } \\
\hline & 1 & 2 & 3 & 4 & 5 \\
\hline \multicolumn{6}{|l|}{ Anacardiaceae } \\
\hline Schinus & 21,9 & 11,1 & 32,2 & 34,8 & 36,1 \\
\hline Spondias & 2,1 & 1,9 & 1,1 & 1,9 & 0,9 \\
\hline \multicolumn{6}{|l|}{ Euphorbiaceae } \\
\hline Croton & 8,1 & 10,8 & 24,4 & 19,9 & 22,8 \\
\hline \multicolumn{6}{|l|}{ Fabaceae } \\
\hline Anadenanthera & 0,6 & 0,3 & 0,8 & 0,3 & 0,3 \\
\hline Caesalpinia & 14,1 & 3,4 & 3,3 & 5,4 & 9,0 \\
\hline Fabaceae 1 & & 2,1 & & & \\
\hline Mimosa tenuiflora & 27,9 & 33,1 & 28,9 & 33,9 & 29,0 \\
\hline Mimosa subenervis & & 0,3 & 0,3 & & \\
\hline Prosopis & 0,3 & & & & \\
\hline Senna macranthera & 0,6 & & 0,6 & 0,3 & \\
\hline \multicolumn{6}{|l|}{ Myrtaceae } \\
\hline Myrcia & 0,3 & 0,8 & & 0,3 & 0,6 \\
\hline \multicolumn{6}{|l|}{ Malvaceae } \\
\hline Pseudobombax & & & 1,7 & 1,6 & 0,3 \\
\hline Waltheria & & 1,3 & & & \\
\hline Sida & & & & & 0,3 \\
\hline \multicolumn{6}{|l|}{ Poaceae } \\
\hline \multicolumn{6}{|l|}{ Poaceae 1} \\
\hline \multicolumn{6}{|l|}{ Solanaceae } \\
\hline Solanum & & & 0,3 & & \\
\hline Indeterminados* & $\begin{array}{c}24,0 \\
\text { (3) } \\
\end{array}$ & $\begin{array}{c}34,9 \\
(1)\end{array}$ & $\begin{array}{l}6,4 \\
(1) \\
\end{array}$ & $\begin{array}{l}1,6 \\
(2)\end{array}$ & $\begin{array}{l}0,6 \\
(1)\end{array}$ \\
\hline
\end{tabular}

*Frequência de tipos polínicos (quantidade de tipos polínicos).

estabilidade durante a estocagem do mel podendo estar diretamente relacionado com a composição florística nas áreas de coleta, além das diferenças na composição do solo ou associação com outras espécies vegetais. ${ }^{20}$

Os valores da acidez variaram entre 85,83 e 103,33 50 meq $\mathrm{kg}^{-1}$ (Tabela 2), sendo acima de $50 \mathrm{meq} \mathrm{kg}^{-1}$ que é o permitido para abelhas sem ferrão. A variação pode ser devido a vários fatores, incluindo o armazenamento das amostras antes das análises, variação dos ácidos orgânicos causada pelas diferentes fontes de néctar, ação das bactérias durante a maturação do mel, quantidade de minerais presentes, entre outros parâmetros não identificados. A acidez pode ser um indicativo de fermentação de açúcares em ácidos orgânicos. ${ }^{21}$
Os valores da atividade de água para as cinco amostras dos méis variaram de 0,70 a 0,76 (Tabela 2), estando dentro das normas publicadas para os méis das abelhas nativas, que é de $0,80 .{ }^{6}$ Para umidade os valores foram de 26 e $27 \%$, permitidos para o mel in natura (mel extraído dos potes e mantido sob refrigeração logo após a sua coleta até o momento do consumo, não submetido a qualquer outro processamento), pasteurizado (mel que após a extração passa por processo térmico de pasteurização para redução e/ou inibição do desenvolvimento microbiológico e/ou da atividade enzimática no produto, sendo posteriormente mantido à temperatura ambiente ou sob refrigeração) ou maturado (mel que após a extração passa pelo processo de maturação em temperatura ambiente, caracterizado por sua fermentação natural, a partir do desenvolvimento das leveduras osmofílicas naturalmente presentes) de abelhas nativas que é no

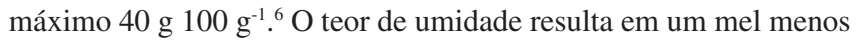
viscoso, sendo uma característica única para os méis das abelhas nativas. A quantidade de água no mel de meliponíneos é considerada o grande diferencial deste produto em relação ao mel de Apis mellifera. ${ }^{22} \mathrm{~A}$ atividade de água é um parâmetro importante para avaliar o estado e a estabilidade relativa, no que diz respeito às propriedades físicas de qualquer alimento, velocidade das reações de alteração e atividade enzimática, assim como crescimento e desenvolvimento de microrganismos. ${ }^{23}$

A análise da condutividade das amostras mostrou valores que variaram de 587,66 a $651,66 \mu \mathrm{S} \mathrm{cm}^{-1}$ (Tabela 2). A condutividade elétrica do mel está diretamente relacionada com a origem floral e depende da concentração dos sais minerais, ácidos orgânicos, proteínas e outras substâncias. ${ }^{24,25}$ De acordo com Yadata, ${ }^{25}$ os méis escuros apresentam maiores valores para a condutividade elétrica, indicando a presença de ácidos e compostos ionizáveis em solução aquosa.

Os valores médios obtidos para os açúcares redutores (frutose e glicose) foram de 59,44 a 60,76/100 g, estando de acordo com os recomendados pela legislação (limite mínimo de 60/100 g). Méis de meliponas possuem menor teor de açúcares $(70 \%)$, sendo mais adocicados. $^{26}$

O teor de hidroxifurfural (HMF) dos méis analisados apresentaram valores bem abaixo do permitido (até $20 \mathrm{mg} \mathrm{kg}^{-1}$ de mel) de 3,04 a 5,23 $\mathrm{mg} \mathrm{kg}^{-1}$ (Tabela 2). A avaliação de HMF é um parâmetro para verificação do frescor e ou/superaquecimento do mel.

$\mathrm{O}$ teor de cinzas nas amostras analisadas variou de 0,18 a $0,26 \%$ (Tabela 2) e está de acordo com a legislação vigente (máximo de $0,6 \%$ ). O teor de cinzas representa a riqueza dos minerais no mel, sendo um parâmetro que é frequentemente utilizado para fins de controle de qualidade. O conteúdo mineral no mel é geralmente pequeno e depende da composição do néctar das espécies vegetais, tipo de solo e está associado a diferentes origens botânicas e geográficas. ${ }^{27}$

Com exceção da acidez, todos os parâmetros físico-químicos para as cinco amostras dos méis de abelha branca apresentaram-se normais de acordo com os dados registrados na literatura e nas legislações para os méis das abelhas nativas sem ferrão.

Tabela 2. Análises físico-químicas do mel de abelha Branca (Frieseomelitta doederleini) ${ }^{\mathrm{a}}$

\begin{tabular}{|c|c|c|c|c|c|c|c|c|}
\hline Amostras & $\mathrm{pH}$ & $\begin{array}{l}\text { Acidez livre } \\
(\text { meq kg-1) }\end{array}$ & $\begin{array}{l}\text { Atividade de } \\
\text { Agua (aw) }\end{array}$ & Umidade & $\begin{array}{l}\text { Condutividade } \\
\qquad\left(\mu \mathrm{S} \mathrm{cm} \mathrm{cm}^{-1}\right)\end{array}$ & $\begin{array}{c}\text { Açúcar redutor } \\
(\%)\end{array}$ & $\operatorname{HMF}\left(\mathrm{mg} \mathrm{kg}^{-1}\right)$ & $\begin{array}{c}\text { Teor de cinzas } \\
(\%)\end{array}$ \\
\hline 1 & $3,68 \pm 0,01$ & $103,33 \pm 1,40$ & $0,75 \pm 0,01$ & $26,83 \pm 0,28$ & $595,33 \pm 0,57$ & $60,76 \pm 0,33$ & $4,49 \pm 0,29$ & $0,25 \pm 0,05$ \\
\hline 2 & $3,79 \pm 0.01$ & $94,16 \pm 1,44$ & $0,73 \pm 0,01$ & $26,66 \pm 0,57$ & $651,66 \pm 0,57$ & $60,56 \pm 0,33$ & $4,13 \pm 0,31$ & $0,22 \pm 0,01$ \\
\hline 3 & $3,82 \pm 0,01$ & $94,16 \pm 1,44$ & $0,76 \pm 0,01$ & $27,33 \pm 0,57$ & $587,66 \pm 0,57$ & $60,00 \pm 0,32$ & $3,04 \pm 0,17$ & $0,18 \pm 0,03$ \\
\hline 4 & $3,92 \pm 0,01$ & $90,00 \pm 2,50$ & $0,75 \pm 0,01$ & $27,33 \pm 0,57$ & $631,33 \pm 0,57$ & $60,00 \pm 0,32$ & $5,23 \pm 0,68$ & $0,20 \pm 0,03$ \\
\hline 5 & $3,91 \pm 0,01$ & $85,83 \pm 1,44$ & $0,70 \pm 0,02$ & $27,46 \pm 0,05$ & $631,33 \pm 0,57$ & $59,44 \pm 0,31$ & $4,74 \pm 0,22$ & $0,26 \pm 0,01$ \\
\hline
\end{tabular}

avalor médio \pm desvio padrão, $\mathrm{n}=3$. 


\section{Teor de minerais nos méis de abelha branca}

O teor dos minerais ( $\mathrm{Na}, \mathrm{K}, \mathrm{Ca}$ e $\mathrm{Mg}$ ) encontrados nos méis de abelha branca estão na Tabela 3. É verificado que o mineral mais abundante é o $\mathrm{K}$ seguido do $\mathrm{Mg}$. A composição mineral do mel varia de acordo com a origem floral, clima, solo e fatores relativos às abelhas. Em méis das abelhas Melipona fasciculata e Melipona flavoneata foi verificado que o mineral mais abundante foi o $\mathrm{K}$, seguido do $\mathrm{Na}, \mathrm{Ca}$ e Mg. ${ }^{28}$ Para amostras de méis monoflorais e heteroflorais coletados pelas abelhas Apis melifera no estado do Ceará, região Nordeste do Brasil foi verificado que entre os minerais detectados nas amostras de méis analisadas, $\mathrm{K}$ apresentou a maior concentração seguido por $\mathrm{Ca}, \mathrm{Na}$, e $\mathrm{Mg} .{ }^{29} \mathrm{O}$ conteúdo mineral é frequentemente expresso em macro e microelementos e sua composição é dependente de condições intrínsecas (origem botânica) e extrínsecas (solo, origem geográfica). Esses bioelementos estão presentes em amostras de meis, estando entre os principais macronutrientes $\mathrm{K}, \mathrm{Ca}, \mathrm{Mg}$ e Na e micronutrientes $\mathrm{Fe}, \mathrm{Cu}, \mathrm{Zn}, \mathrm{Mn}$ e Se. ${ }^{29,30}$ Os minerais são essenciais para regulação das vias metabólicas e processos fisiológicos. Sua ingestão adequada é fundamental para manter a homeostase, proteção celular, funcionalidade e saúde, enquanto a sua deficiência pode desencadear doenças específicas. De acordo com a regulamentação brasileira ${ }^{31}$ para minerais, a contribuição de mais de $30 \%$ da IDR (ingestão diária recomendada) de referência por $100 \mathrm{~g}$ de produto, classifica o alimento como "alto conteúdo" e acima de $15 \%$ como "fonte". Com base na IDR, somente o potássio presente nas amostras dos méis de abelha branca pode ser considerado como "fonte" desse mineral.

\section{Análises por UPLC-PDA-qTOF-MS/MS}

Alguns compostos conhecidos presentes no mel de abelha branca foram identificados por comparação com padrões autênticos de acordo com os tempos de retenção, dados de ultravioleta (UV) e espectros de massas. Os compostos não conhecidos foram caracterizados pela análise dos íons produtos (espectros $\mathrm{MS}^{\mathrm{E}}$ ) e pelos espectros de UV comparados com registros na literatura. Dos 34 compostos analisados nas amostras de méis, trinta e dois deles foram identificados (Tabela 4), desses, os compostos 1, 7, 8, 12, 13 e 30 foram comparados com padrões autênticos. Com exceção dos compostos $\mathbf{3}$ e $\mathbf{4}$ (não foi possível a identificação somente com os espectros de massas) todos são derivados fenólicos. Apenas três compostos não são flavonoides agliconas: 1 (ácido elágico), $\mathbf{2}$ (dihidroxicumarina) e $\mathbf{5}$ (isoramnetina-glicosídeo). Dos 29 flavonóides agliconas identificados 21 são flavonas/flavonois e 8 são flavanonas/flavanonois. Os cromatogramas obtidos por UPLC-DAD e ion pico base (UPLC-qTOF/MS ${ }^{\mathrm{E}}$ ) em modo negativo da fração SPE metanólica do mel de abelha branca (amostra 3) estão nas Figuras 1 e 2, respectivamente.

O pico 1 mostrou o íon [M-H] em $\mathrm{m} / z, 300.9997$ e foi identificado como ácido elágico. Os íons produtos em $\mathrm{m} / \mathrm{z} 283.9943$ $\left[\mathrm{M}-\mathrm{H}_{2} \mathrm{O}\right]^{-}, m / z 257.0072\left[\mathrm{M}-\mathrm{H}-\mathrm{CO}_{2}\right]^{-}, m / z 229.9945\left[\mathrm{M}-\mathrm{H}-\mathrm{CO}_{2}-\mathrm{CO}\right]^{-}$,

Tabela 3. Teor de minerais $\left(\mathrm{mg} \mathrm{kg}^{-1}\right)$ no mel de abelha Branca (Frieseomelitta doederleini) ${ }^{\mathrm{a}}$

\begin{tabular}{ccccc}
\hline Amostra de mel & $\mathrm{K}$ & $\mathrm{Mg}$ & $\mathrm{Na}$ & $\mathrm{Ca}$ \\
\hline 1 & $1259,60 \pm 16,90$ & $40,31 \pm 0,56$ & $11,81 \pm 0,50$ & $6,58 \pm 0,50$ \\
2 & $1260,98 \pm 33,57$ & $40,23 \pm 0,66$ & $9,92 \pm 0,49$ & $6,21 \pm 0,43$ \\
3 & $1287,41 \pm 38,04$ & $35,98 \pm 0,81$ & $12,66 \pm 0,28$ & $5,16 \pm 0,44$ \\
4 & $1145,73 \pm 8,53$ & $35,19 \pm 0,56$ & $17,13 \pm 1,92$ & $5,58 \pm 0,59$ \\
5 & $1334,07 \pm 37,64$ & $40,22 \pm 0,46$ & $13,20 \pm 1,54$ & $5,50 \pm 0,43$ \\
\hline
\end{tabular}

${ }^{\mathrm{a}}$ valor médio \pm desvio padrão, $\mathrm{n}=3$.

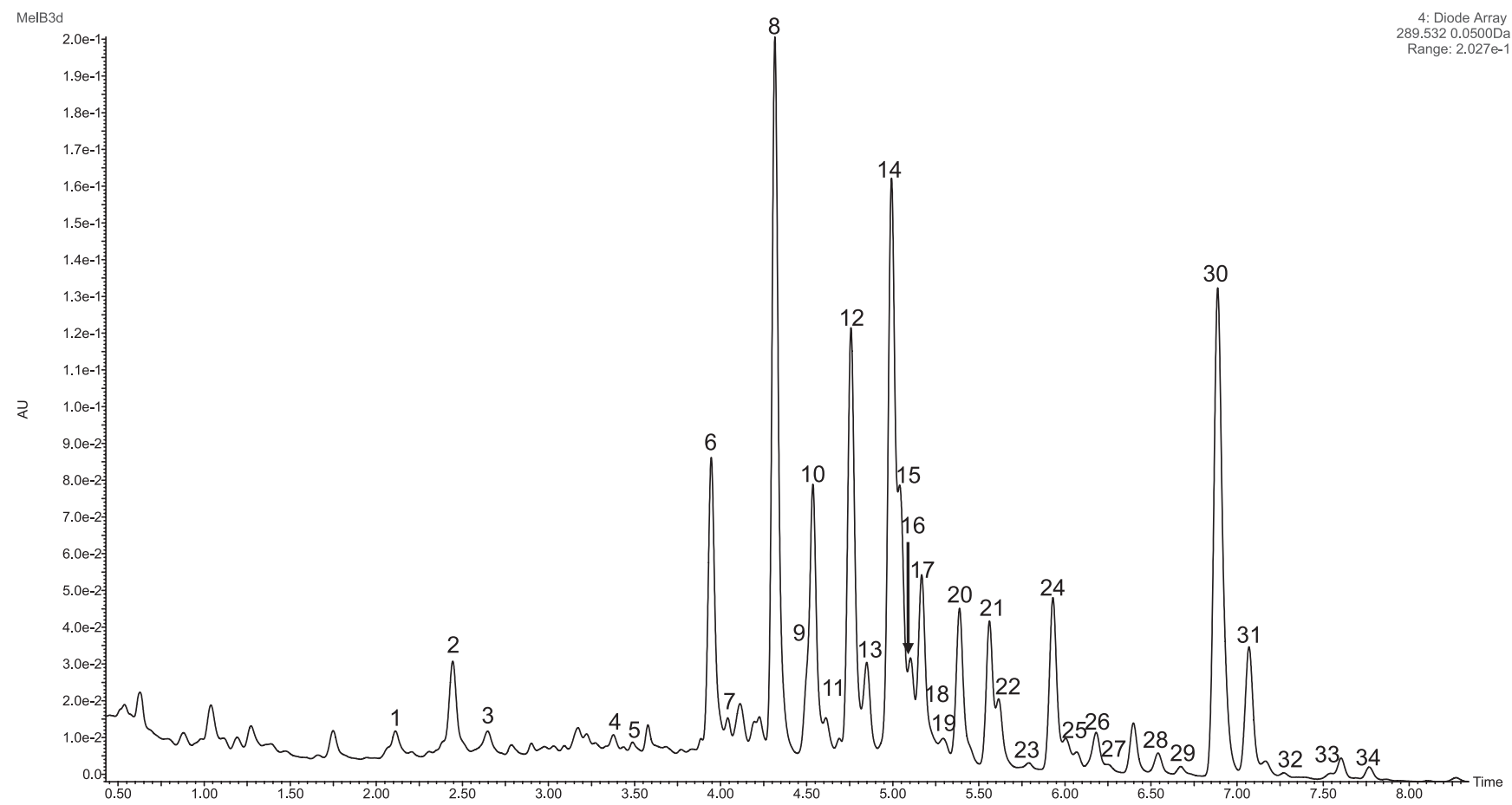

Figura 1. UPLC-DAD (290 nm) da fração SPE metanólica (amostra 3) do mel de abelha branca 


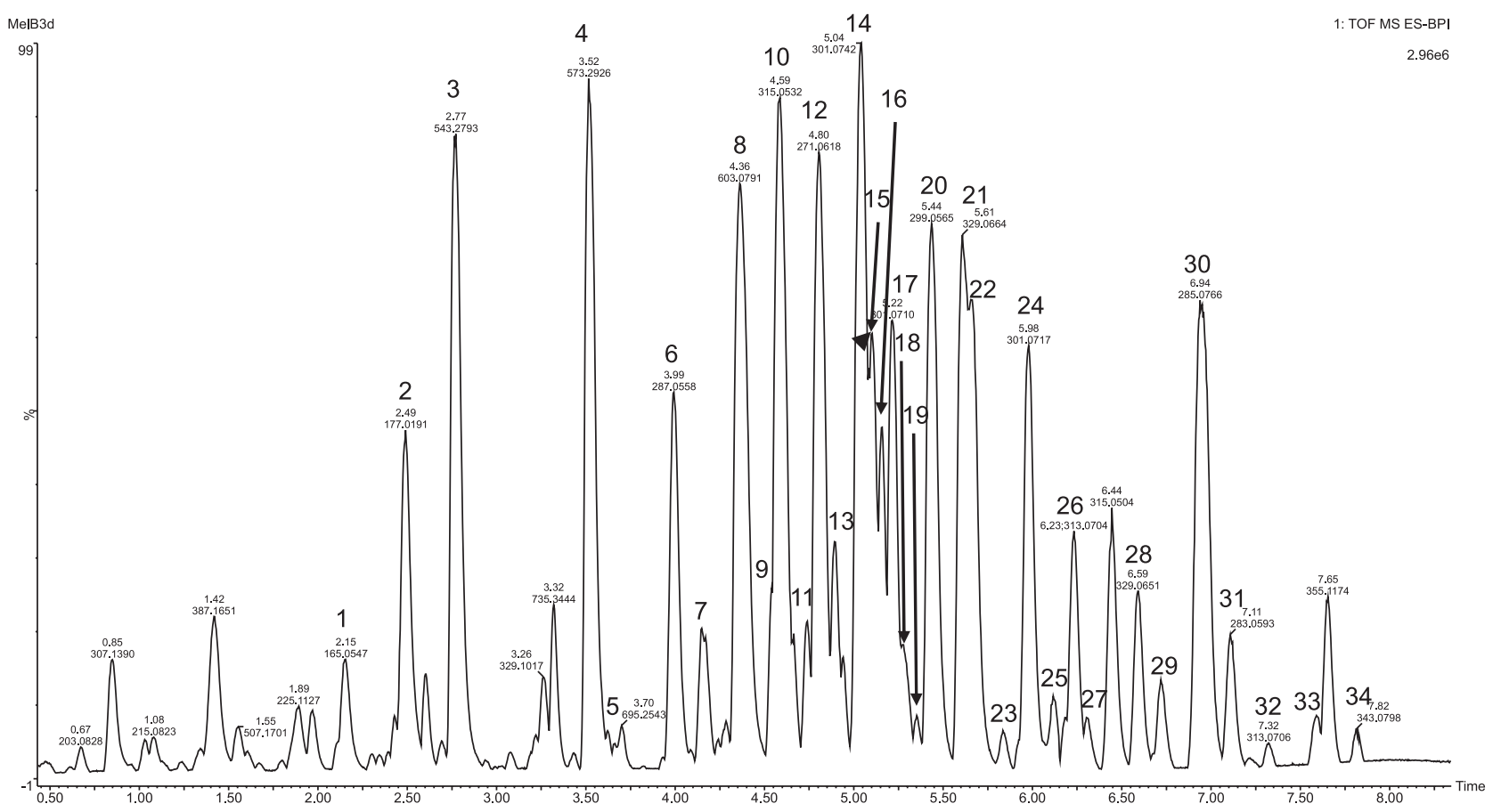

Figura 2. Cromatograma do ín pico base obtido por $M S^{E}\left(U P L C-q T O F / M S^{E}\right)$ em modo negativo da fração SPE metanólica (amostra 3) do mel de abelha branca

$m / z 201.0184\left[\mathrm{M}-\mathrm{H}-\mathrm{CO}_{2}-2 \mathrm{CO}\right]^{-}$, and $\mathrm{m} / z, 185.0241\left[\mathrm{M}-\mathrm{H}-2 \mathrm{CO}_{2}{ }^{-}\right.$ $\mathrm{CO}]^{-}$corroboram com a identificação.32 O pico 2 apresentou o íon [M-H] em $\mathrm{m} / \mathrm{z}$ 177,0193 [M-H]. Os íons produtos em $\mathrm{m} / \mathrm{z}$ 133, 0292 e 89,0383 são referentes à perda de $\mathrm{CO}_{2}$ e em $\mathrm{m} / \mathrm{z}$. 105,0334 é devido a perda de CO. Esses dados sugerem que o composto 2 pode ser uma cumarina dihidroxilada. ${ }^{33}$ Os compostos $\mathbf{3}$ e $\mathbf{4}$ não foram identificados com os dados obtidos pelos espectros de massas e não apresentam absorção no UV. Composto 5 (isoramnetina-glicosídeo) $\operatorname{com} \mathrm{m} / \mathrm{z}, 477,2039$ [M-H] $^{-}$mostrou a perda de $162 \mathrm{Da}$, sugerindo a presença de uma hexose na estrutura. O fragmento em $\mathrm{m} / 2,315.0502$ [M-H-hexose $]^{-}$corresponde a molécula da isoramnetina (quercetina metilada) desprotonada.

Os flavonoides agliconas foram separadas em dois grupos baseados nos valores das absorções no UV (em torno de $290 \mathrm{~nm}$ para flavanona/flavanonol e 345-360 nm para flavona/flavonol) e nos íons produtos obtidos nos espectros de massas, sendo a grande maioria isômeros como: 7, 30 (285 Da); 8, 14, 15, 17, 24 (301 da); 10, 16, 18, 25 (315 Da); 11, 19 (345 Da); 21, 22, 27, 28 (329 Da); 26, 32, 33 (313 Da) e 29, 34 (343 Da). Os picos mais comuns derivados da perda neutra de pequenas moléculas ou íons como por exemplo $\mathrm{CO}$ (28 Da), $\mathrm{H}_{2} \mathrm{O}$ (18 Da) ou $\mathrm{CH}_{3}(15 \mathrm{Da})$ estão na Tabela 4. Geralmente a presença dos íons produtos resultantes da perda dessas moléculas com baixa intensidade e a presença acentuada do pico do íon desprotonado é observado no espectro $\mathrm{MS}^{\mathrm{E}}$ obtido com a mais baixa energia de colisão. No mesmo tipo de análise $\mathrm{MS}^{\mathrm{E}}$ utilizando-se uma rampa com maior energia de colisão que varia de 10 a $30 \mathrm{~V}$, a abundância relativa dos íons produtos que geram moléculas neutras é aumentada, como também aparecem outros fragmentos resultantes da quebra do anel $\mathrm{C}$ dos flavonoides, clivagem retro Diels-Alder (RDA), pois com o aumento da energia de colisão, ocorre uma completa fragmentação a partir da molécula desprotonada. A clivagem RDA resulta em fragmentos que revelam o padrão de substituição nos anéis A e B. Os íons produtos são denominados ${ }^{\mathrm{i}, \mathrm{j}} \mathrm{A}-\mathrm{e}^{\mathrm{i}, \mathrm{j}} \mathrm{B}-, \mathrm{A}$ e $\mathrm{B}$ representam os anéis e os números sobrescritos indicam a quebra das ligações a partir das moléculas desprotonadas. ${ }^{34,35}$ Desse modo, os fragmentos representativos das clivagens das ligações 0,2 e 1,3 do anel C em RDA são ${ }^{0,2} \mathrm{~A}$; ${ }^{0,2} \mathrm{~B}^{-} \mathrm{e}^{1,3} \mathrm{~A}^{-},{ }^{1,3} \mathrm{~B}^{-}$, respectivamente. A quebra através da retrociclização do anel $\mathrm{C}$ que representa as duas classes dos flavonoides está na Figura 3 e os íons produtos $\left(\mathrm{MS}^{2}\right)$ obtidos para todos os flavonoides identificados estão na Tabela 5. Os fragmentos obtidos resultantes do anel A e B indicam um possível padrão de substituição nestes anéis relativo ao número de hidroxilas e metoxilas presentes, embora não possa indicar a posição exata destes grupos no anel. Um exemplo para as duas classes de flavonoides detectados está na Figura 3, na qual são mostrados os principais íons produtos para a isoramnetina e 7-metoxi-naringenina, respectivamente. Isoramnetina representa um flavonol que apresenta o íon desprotonado em $\mathrm{m} / \mathrm{z}$ 315,0506 [M-H] ${ }^{-}$. $\mathrm{O}$ espectro de massas mostra a perda de metila em $\mathrm{m} / \mathrm{z}, 300,0285$ [M-H-CH $]_{3}$, perda de água 283,0275 [M-2H-CH$\left.-\mathrm{CH}_{3}-\mathrm{H}_{2}\right]^{-}$e subsequentes perdas de $\mathrm{CO} \mathrm{m} / \mathrm{z}$ 271,0248 [M-2 $\left.\mathrm{H}-\mathrm{CH}_{3}-\mathrm{CO}\right]$;, 255,0310 $\left[\mathrm{M}-2 \mathrm{H}-\mathrm{CH}_{3}-\mathrm{H}_{2} \mathrm{O}-\mathrm{CO}\right]^{-}$e $\mathrm{m} / z, 227,0358\left[\mathrm{M}-2 \mathrm{H}-\mathrm{CH}_{3}-\mathrm{H}_{2} \mathrm{O}-2 \mathrm{CO}\right]^{-}$. De acordo com Huck et al. ${ }^{36}$ as flavonas metoxiladas primeiro perdem a metila [M-H- $\left.\mathrm{CH}_{3}\right]^{-}$, depois ocorre desidratação [M-2H-CH$\left.-\mathrm{CH}_{2} \mathrm{O}\right]^{-} \mathrm{e}$ perda de carbonila. A clivagem do anel $\mathrm{C}$ referente a RDA forneceu os íons produtos em $\mathrm{m} / \mathrm{z}, 164,0111, \mathrm{~m} / \mathrm{z}, 149,9961, \mathrm{~m} / \mathrm{z}, 151,0025$ referentes aos fragmentos ${ }^{0,2} \mathrm{~A}^{-},{ }^{0,2} \mathrm{~B}^{-} \mathrm{e}^{1,3} \mathrm{~A}^{-}$, respectivamente. $\mathrm{O}$ íon produto em $\mathrm{m} / \mathrm{z}, 164,0111$ demonstra que a metoxila está ligada no anel $\mathrm{B}$ da isoramnetina. Os flavonoides semelhantes à isoramnetina apresentaram perfil de ionização similares (Tabela 4). A 7-metoxi-naringenina, representante de uma flavanona, apresentou o pico do íon desprotonado em $\mathrm{m} / z$ 285,0762 [M-H] $]^{-}$. Os principais íons produtos formados foram $\mathrm{m} / \mathrm{z}$ 269,0383 [M-2H- $\left.\mathrm{CH}_{3}\right]^{-}$e $\mathrm{m} / \mathrm{z}, 252,0089$ [M-2H-CH $\left.-\mathrm{H}_{2} \mathrm{O}\right]^{-}$, além dos íons referentes a RDA em $\mathrm{m} / z$, 165,0467 $\left({ }^{0,2} \mathrm{~A}^{-}\right)$e $m / z, 119,0494\left({ }^{0,2} \mathrm{~B}^{-}\right)$. Mais uma vez a presença da metoxila na posição 7 do anel A foi confirmada pelo pico referente $\mathrm{a}^{0,2} \mathrm{~A}^{-} \mathrm{de}$ $\mathrm{m} / \mathrm{z}$ 165,0467 (Figura 3). Os picos dos íons produtos obtidos de todas as outras flavanonas ou flavanonois estão nas Tabelas 4 e 5 .

Uma vez que a análise melissopalinológica mostrou uma maior porcentagem de pólens coletados da espécie Mimosa tenuiflora (Tabela 1) com o indicativo que as abelhas visitaram esta espécie vegetal com maior frequência, as flores de Mimosa tenuiflora (jurema preta) foram coletadas para extração e análise por UPLC-DADqTOF-MS/MS e os cromatogramas estão na Figura 4. As análises dos cromatogramas mostraram que todos os flavonoides (compostos 5-34) 
Tabela 4. Identificação dos compostos dos méis de abelha branca por UPLC-DAD-QTOF-MS-MS

\begin{tabular}{|c|c|c|c|c|c|c|}
\hline & $\mathrm{RT}(\min )$ & $\lambda_{\max }(\mathrm{nm})$ & {$[\mathrm{M}-\mathrm{H}]^{-}(m / z)$} & $\begin{array}{c}{[\mathrm{M}-\mathrm{H}]^{-}(\mathrm{m} / \mathrm{z})} \\
\text { Calculado }\end{array}$ & & $\begin{array}{l}\text { Tentativa de } \\
\text { identificação }\end{array}$ \\
\hline 1 & 2,15 & 364 & 300,9997 & 300,9990 & $\begin{array}{c}257,0072\left[\mathrm{M}-\mathrm{H}-\mathrm{CO}_{2}\right]^{-}, 229,9945\left[\mathrm{M}-\mathrm{H}_{-}-\mathrm{CO}_{2}-\mathrm{CO}\right]^{-}, \\
185,0241\left[\mathrm{M}-\mathrm{H}-\mathrm{CO}_{2}-\mathrm{CO}\right]^{-}\end{array}$ & Ácido elágico* \\
\hline 2 & 2,49 & 293 & 177,0193 & 177,0193 & $133,0292\left[\mathrm{M}-\mathrm{H}-\mathrm{CO}_{2}\right]^{-}, 105,0334[\mathrm{M}-\mathrm{H}-\mathrm{CO}]^{-}, 89,0383\left[\mathrm{M}-\mathrm{H}-2 \mathrm{CO}_{2}\right]^{-}$ & Dihidroxicumarina \\
\hline 3 & 2,77 & - & 543,2815 & - & & Não identificado \\
\hline 4 & 3,52 & - & 573,2867 & - & & Não identificado \\
\hline 5 & 3,65 & 345 & 477,1039 & 477,1039 & $315,0502[\mathrm{M}-\mathrm{H} \text {-glicose }]^{-}$ & $\begin{array}{l}\text { Isoramnetina- } \\
\text { glicosídeo }\end{array}$ \\
\hline$\underline{6}$ & 3,99 & 287 & 287,0558 & 287,0561 & 259,9099 [M-H-CO] $^{-}$ & Tetrahidroxi-flavanona \\
\hline 7 & 4,15 & 359 & 285,0400 & 285,0405 & $257,0378[\mathrm{M}-\mathrm{H}-\mathrm{CO}]^{-}$ & Luteolina* \\
\hline 8 & 4,36 & 345 & 301,0305 & 301,0353 & $283,0178\left[\mathrm{M}-\mathrm{H}-\mathrm{H}_{2} \mathrm{O}\right]^{-}, 273,0399[\mathrm{M}-\mathrm{H}-\mathrm{CO}]^{-}$ & Quercetina* \\
\hline 9 & 4,52 & 345 & 331,0437 & 331,0459 & $\begin{array}{c}316,0200\left[\mathrm{M}-\mathrm{H}-\mathrm{CH}_{3}\right]^{-}, 301,0304\left[\mathrm{M}-\mathrm{H}-2 \mathrm{CH}_{3}\right]^{-} \\
272,0483\left[\mathrm{M}-2 \mathrm{H}-2 \mathrm{CH}_{3}-\mathrm{CO}\right]^{-}\end{array}$ & $\begin{array}{l}\text { Pentahidroxi-metoxi- } \\
\text { flavona }\end{array}$ \\
\hline 10 & 4,59 & 356 & 315,0576 & 315,0510 & $\begin{array}{c}300,0258\left[\mathrm{M}-\mathrm{H}-\mathrm{CH}_{3}\right]^{-}, 271,0230\left[\mathrm{M}-2 \mathrm{H}^{\left.-\mathrm{CH}_{3}-\mathrm{CO}\right]^{-}}\right. \\
272,0288\left[\mathrm{M}-\mathrm{H}-\mathrm{CH}_{3}-\mathrm{CO}\right]^{-}, 255,0301\left[\mathrm{M}-2 \mathrm{H}^{-} \mathrm{CH}_{3}-\mathrm{CO}-\mathrm{H}_{2} \mathrm{O}\right]^{-}\end{array}$ & $\begin{array}{l}\text { Tetra-hidroxi-metoxi- } \\
\text { flavona }\end{array}$ \\
\hline 11 & 4,74 & 358 & 345,0608 & 345,0608 & $\begin{array}{c}330,0374\left[\mathrm{M}-\mathrm{H}-\mathrm{CH}_{3}\right]^{-}, 315,0137\left[\mathrm{M}-\mathrm{H}-2 \mathrm{CH}_{3}\right]^{-} \\
287,0194\left[\mathrm{M}-\mathrm{H}-2 \mathrm{CH}_{3}-\mathrm{CO}\right]^{-}\end{array}$ & $\begin{array}{c}\text { Pentahidroxi- } \\
\text { dimetoxi-flavona }\end{array}$ \\
\hline 12 & 4,80 & 288 & 271,0606 & 271,0611 & $253,0455\left[\mathrm{M}-\mathrm{H}-\mathrm{H}_{2} \mathrm{O}\right]^{-}$ & Naringerina* \\
\hline 13 & 4,89 & 345 & 269,0451 & 269,0455 & 241,3104 [M-H-CO] $^{-}$ & Apigenina* \\
\hline$\overline{14}$ & 5,04 & 290 & 301,0790 & 301,0716 & $286,0473\left[\mathrm{M}-\mathrm{H}-\mathrm{CH}_{3}\right]^{-}$ & $\begin{array}{l}\text { Trihidroxi-metoxi- } \\
\text { flavanona }\end{array}$ \\
\hline$\overline{15}$ & 5,10 & 288 & 301,0710 & 301,0716 & $285,0385\left[\mathrm{M}-2 \mathrm{H}-\mathrm{CH}_{3}\right]^{-}$ & $\begin{array}{l}\text { Trihidroxi-metoxi- } \\
\text { flavanona }\end{array}$ \\
\hline 16 & 5,16 & 360 & 315,0505 & 315,0510 & 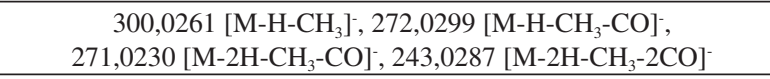 & $\begin{array}{l}\text { Tetrahidroxi-metoxi- } \\
\text { flavona } \\
\end{array}$ \\
\hline 17 & 5,22 & 286 & 301,0701 & 301,0716 & $286,0464\left[\mathrm{M}-\mathrm{H}-\mathrm{CH}_{3}\right]^{-}$ & $\begin{array}{l}\text { Trihidroxi-metoxi- } \\
\text { flavanona }\end{array}$ \\
\hline 18 & 5,28 & 345 & 315,0506 & 315,0510 & 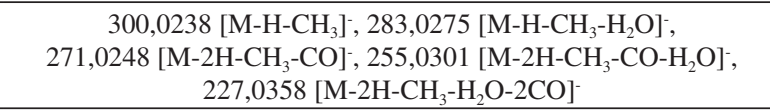 & Isoramnetina* \\
\hline
\end{tabular}

\begin{tabular}{|c|c|c|c|c|c|c|}
\hline 19 & 5,35 & 346 & 329,0638 & 329,0666 & $\begin{array}{c}314,0409\left[\mathrm{M}-\mathrm{H}-\mathrm{CH}_{3}\right]^{,}, 299,0177\left[\mathrm{M}-\mathrm{H}-2 \mathrm{CH}_{3}\right]^{-} \\
271,0231\left[\mathrm{M}-\mathrm{H}-2 \mathrm{CH}_{3}-\mathrm{CO}\right]^{-}\end{array}$ & $\begin{array}{l}\text { Tetrahidroxi-dimetoxi- } \\
\text { flavona }\end{array}$ \\
\hline 20 & 5,44 & 349 & 299,0558 & 299,0561 & $\begin{array}{c}284,0302\left[\mathrm{M}-\mathrm{H}-\mathrm{CH}_{3}\right]^{-}, 255,0276\left[\mathrm{M}-2 \mathrm{H}^{-} \mathrm{CH}_{3}-\mathrm{CO}\right]^{-} \\
227,0328\left[\mathrm{M}-2 \mathrm{H}-\mathrm{CH}_{3}-2 \mathrm{CO}\right]^{-}\end{array}$ & $\begin{array}{l}\text { Trihidroxi-metoxi- } \\
\text { flavona }\end{array}$ \\
\hline 21 & 5,61 & 339 & 329,0663 & 329,0666 & $\begin{array}{c}314,0415\left[\mathrm{M}-\mathrm{H}-\mathrm{CH}_{3}\right]^{-}, 299,0180\left[\mathrm{M}-\mathrm{H}-2 \mathrm{CH}_{3}\right]^{-} \\
271,0232\left[\mathrm{M}-\mathrm{H}-2 \mathrm{CH}_{3}-\mathrm{CO}\right]^{-}\end{array}$ & $\begin{array}{l}\text { Trihidroxi-dimetoxi- } \\
\text { flavona }\end{array}$ \\
\hline 22 & 5,67 & 355 & 329,0663 & 329,0666 & $\begin{array}{c}314,0394\left[\mathrm{M}-\mathrm{H}-\mathrm{CH}_{3}\right]^{-}, 299,0163\left[\mathrm{M}-\mathrm{H}-2 \mathrm{CH}_{3}\right]^{-} \\
271,0261\left[\mathrm{M}-\mathrm{H}-2 \mathrm{CH}_{3}-\mathrm{CO}\right], 243,0272\end{array}$ & $\begin{array}{l}\text { Trihidroxi-dimetoxi- } \\
\text { flavona }\end{array}$ \\
\hline 23 & 5,84 & 346 & 359,0772 & 359,0772 & $\begin{array}{c}344,0544\left[\mathrm{M}-\mathrm{H}_{-} \mathrm{CH}_{3}\right]^{-}, 329,0302\left[\mathrm{M}-\mathrm{H}-2 \mathrm{CH}_{3}\right]^{-}, \\
301,0359\left[\mathrm{M}-\mathrm{H}-2 \mathrm{CH}_{3}-\mathrm{CO}\right]^{-}, 286,0110\left[\mathrm{M}-\mathrm{H}-3 \mathrm{CH}_{3}-\mathrm{CO}\right]^{-}\end{array}$ & $\begin{array}{l}\text { Trihidroxi-trimetoxi- } \\
\text { flavona }\end{array}$ \\
\hline 24 & 5,98 & 286 & 301,0716 & 301,0716 & $283,0501\left[^{\left.\mathrm{M}-\mathrm{H}-\mathrm{H}_{2} \mathrm{O}\right]^{-}}\right.$ & $\begin{array}{l}\text { Trihidroxi-metoxi- } \\
\text { flavanona }\end{array}$ \\
\hline 25 & 6,12 & 287 & 315,0878 & 315,0874 & $285,0410\left[\mathrm{M}-\mathrm{H}-2 \mathrm{CH}_{3}\right]^{-}$ & $\begin{array}{l}\text { Dihidroxi-metoxi- } \\
\text { flavanona }\end{array}$ \\
\hline 26 & 6,23 & 333 & 313,0714 & 313,0717 & $\begin{array}{c}298,0471\left[\mathrm{M}-\mathrm{H}-\mathrm{CH}_{3}\right]^{-}, 283,0235\left[\mathrm{M}-\mathrm{H}-2 \mathrm{CH}_{3}\right]^{-}, \\
255,0291\left[\mathrm{M}-\mathrm{H}-2 \mathrm{CH}_{3}-\mathrm{CO}\right]^{-}\end{array}$ & $\begin{array}{l}\text { Dihidroxi-dimetoxi- } \\
\text { flavona }\end{array}$ \\
\hline 27 & 6,30 & 359 & 329,0666 & 329,0666 & $\begin{array}{c}314,0435\left[\mathrm{M}-\mathrm{H}-\mathrm{CH}_{3}\right]^{-}, 299,0202\left[\mathrm{M}-\mathrm{H}-2 \mathrm{CH}_{3}\right]^{-} \\
271,0266\left[\mathrm{M}-\mathrm{H}-2 \mathrm{CH}_{3}-\mathrm{CO}\right]^{-}\end{array}$ & $\begin{array}{l}\text { Trihidroxi-dimetoxi- } \\
\text { flavona }\end{array}$ \\
\hline 28 & 6,59 & 357 & 329,0655 & 329,0666 & $\begin{array}{c}314,0415\left[\mathrm{M}-\mathrm{H}-\mathrm{CH}_{3}\right]^{-}, 299,0178\left[\mathrm{M}-\mathrm{H}-2 \mathrm{CH}_{3}\right]^{-} \\
271,0234\left[\mathrm{M}-\mathrm{H}-2 \mathrm{CH}_{3}-\mathrm{CO}\right]^{-}\end{array}$ & $\begin{array}{l}\text { Trihidroxi-dimetoxi- } \\
\text { flavona }\end{array}$ \\
\hline 29 & 6,72 & 245 & 343,0808 & 343,0823 & $\begin{array}{c}328,0567\left[\mathrm{M}-\mathrm{H}-\mathrm{CH}_{3}\right]^{-}, 313,0329\left[\mathrm{M}-\mathrm{H}-2 \mathrm{CH}_{3}\right]^{-}, \\
298,0099\left[\mathrm{M}-\mathrm{H}-3 \mathrm{CH}_{3}\right]^{-}\end{array}$ & $\begin{array}{l}\text { Dihidroxi-trimetoxi- } \\
\text { flavona }\end{array}$ \\
\hline 30 & 6,94 & 287 & 285,0762 & 285,0768 & $269,0383\left[\mathrm{M}-2 \mathrm{H}-\mathrm{CH}_{3}\right]^{-}$ & 7-metoxi-naringenina* \\
\hline 31 & 7,11 & 345 & 283,0610 & 283,0611 & $268,0362\left[\mathrm{M}-\mathrm{H}-\mathrm{CH}_{3}\right]^{-}$ & $\begin{array}{l}\text { Dihidroxi-metoxi- } \\
\text { flavona }\end{array}$ \\
\hline 32 & 7,32 & 346 & 313,0714 & 313,0718 & $\begin{array}{c}298,0453\left[\mathrm{M}-\mathrm{H}-\mathrm{CH}_{3}\right]^{-}, 283,0211\left[\mathrm{M}-\mathrm{H}-2 \mathrm{CH}_{3}\right]^{-}, \\
255,0273\left[\mathrm{M}-\mathrm{H}-2 \mathrm{CH}_{3}-\mathrm{CO}\right]^{-}\end{array}$ & $\begin{array}{l}\text { Dihidroxi-dimetoxi- } \\
\text { flavona }\end{array}$ \\
\hline 33 & 7,59 & 357 & 313,0694 & 313,0718 & $\begin{array}{c}298,0453\left[\mathrm{M}-\mathrm{H}-\mathrm{CH}_{3}\right]^{-}, 283,0211\left[\mathrm{M}-\mathrm{H}-2 \mathrm{CH}_{3}\right]^{-}, \\
255,0267\left[\mathrm{M}-\mathrm{H}-2 \mathrm{CH}_{3}-\mathrm{CO}\right]^{-} \\
\end{array}$ & $\begin{array}{l}\text { Dihidroxi-dimetoxi- } \\
\text { flavona }\end{array}$ \\
\hline 34 & 7,82 & 345 & 343,0811 & 343,0813 & $\begin{array}{c}328,0560\left[\mathrm{M}-\mathrm{H}-\mathrm{CH}_{3}\right]^{-}, 313,0325\left[\mathrm{M}-\mathrm{H}-2 \mathrm{CH}_{3}\right]^{-}, \\
285,0378\left[\mathrm{M}-\mathrm{H}-2 \mathrm{CH}_{3}-\mathrm{CO}\right]^{-}, 270,0139\left[\mathrm{M}-\mathrm{H}-3 \mathrm{CH}_{3}-\mathrm{CO}\right]^{-}\end{array}$ & $\begin{array}{l}\text { Hidroxi-trimetoxi- } \\
\text { flavona }\end{array}$ \\
\hline
\end{tabular}


Tabela 5. Íons produtos $\left(\mathrm{MS}^{2}\right)$ dos flavonóides oriundos da retrociclização do anel C (RDA)

\begin{tabular}{|c|c|c|c|c|c|c|c|c|c|}
\hline & {$[\mathrm{M}-\mathrm{H}]^{-}$} & Flavonoides & ${ }^{0,2} \mathrm{~A}^{-}$ & ${ }^{0,2} \mathrm{~B}^{-}$ & ${ }^{0,4} \mathrm{~B}^{-}$ & ${ }^{1,3} \mathrm{~A}^{-}$ & ${ }^{1,3} \mathrm{~B}^{-}$ & {$[\mathrm{M}-\mathrm{H}-\mathrm{B}]^{-}$} & {$\left[\mathrm{M}-\mathrm{H}-\mathrm{B}-\mathrm{CH}_{3}\right]$} \\
\hline 6 & 287,0558 & Tetrahidroxi-flavanona & & 135,0444 & & 151,0029 & & & \\
\hline 7 & 285,0400 & Luteolina & & & & 151,0029 & 133,0291 & 193,0128 & \\
\hline 8 & 301,0305 & Quercetina & & & & 151,0036 & 133,0266 & & \\
\hline 9 & 331,0437 & Pentahidroxi-metoxi-flavona & & & & & & 223,0232 & 208,0002 \\
\hline 10 & 315,0576 & Tetrahidroxi-metoxi-flavona & & & & 151,0057 & 148,0146 & & \\
\hline 11 & 345,0608 & Pentahidroxi-dimetoxi-flavona & & & 175,0050 & & & & \\
\hline 12 & 271,0606 & Naringerina & & 119,0494 & & 151,0027 & & 177,0188 & \\
\hline 13 & 269,0451 & Apigenina & & 119,0499 & & 151,0031 & & & \\
\hline 14 & 301,0790 & Trihidroxi-metoxi-flavanona & 165,9903 & 119,0496 & & 151,0935 & & & \\
\hline 15 & 301,0710 & Trihidroxi-metoxi-flavanona & & & & 151,0031 & & 177,0190 & \\
\hline 16 & 315,0505 & Tetrahidroxi-metoxi-flavona & & & & 165,9903 & & & \\
\hline 17 & 301,0701 & Trihidroxi-metoxi-flavanona & & & & 151,0023 & & & \\
\hline 18 & 315,0506 & Isoramnetina & 163,0033 & 149,0207 & & 151,0029 & 162,9986 & & \\
\hline 19 & 329,0638 & Trihidroxi-dimetoxi-flavona & & & & 151,0029 & & 193,0130 & \\
\hline 20 & 299,0558 & Trihidroxi-metoxi-flavona & 183,0439 & & & & & & \\
\hline 21 & 329,0663 & Trihidroxi-dimetoxi-flavona & 165,9888 & & & & & & \\
\hline 22 & 329,0663 & Trihidroxi-dimetoxi-flavona & & & & & & 193,0482 & \\
\hline 23 & 359,0772 & Trihidroxi-trimetoxi-flavona & 195,8101 & & & & & & \\
\hline 24 & 301,0716 & Trihidroxi-metoxi-flavanona & 165,0181 & & & & & & 135,0441 \\
\hline 25 & 315,0878 & Dihidroxi-metoxi-flavanona & 165,0184 & & & & & & \\
\hline 26 & 313,0714 & Dihidroxi-dimetoxi-flavona & 163,0037 & & & 151,0030 & & & \\
\hline 27 & 329,0666 & Trihidroxi-dimetoxi-flavona & 199,0377 & & & & & & \\
\hline 28 & 329,0655 & Trihidroxi-dimetoxi-flavona & 181,0505 & & & & & & \\
\hline 29 & 343,0808 & Dihidroxi-trimetoxi-flavona & 197,0455 & & & & & & \\
\hline 30 & 285,0762 & 7-metoxi-naringenina* & 177,0483 & 119,0772 & & 165,0466 & 119,0772 & & \\
\hline 31 & 283,0610 & Dihidroxi-metoxi-flavona & 151,0031 & & & & & & \\
\hline 32 & 313,0714 & Dihidroxi-dimetoxi-flavona & & & & & & & \\
\hline 34 & 313,0694 & Dihidroxi-dimetoxi-flavona & 183,0424 & & & & & & \\
\hline 35 & 343,0811 & Hidroxi-trimetoxi-flavona & & & & & & 193,0808 & \\
\hline
\end{tabular}

presentes nos méis de abelha branca são os principais constituintes das flores da jurema preta, ou seja, em relação a esses compostos tanto os méis como a flor de jurema preta apresentam o mesmo perfil químico. Esses dados reforçam que os méis analisados apresentam a maior constituição do néctar coletado das flores desta espécie vegetal como mostrado na analise palinológica. Mais uma vez os dados obtidos neste trabalho mostram o forrageamento específico das abelhas nativas em espécies vegetais também nativas da caatinga brasileira, ${ }^{13}$ indicando que os meliponíneos não apenas interferem nos aspectos sociais e econômicos, mas também em processos ecológicos e no ecossistema. Nesse sentido, existe a necessidade de preservação para a manutenção da diversidade vegetal e da flora nativa aumentando os sítios de nidificação para as abelhas sem ferrão e manutenção do equilíbrio na região semiárida do Nordeste.

Teor de flavonoides, fenólicos totais e atividade antirradicalar (DPPD e ABTS) dos méis da abelha branca (Frieseomelitta doederleini)

Os resultados das análises teor de flavonoides, fenólicos totais e atividades antirradicalares frente aos radicais DPPD e ABTS dos méis da abelha branca estão na Tabela 6. Os testes foram realizados com as amostras de mel in natura. O teor de compostos fenólicos variou de 1,15-1,23 miligramas equivalente ao ácido gálico por grama do mel (mgGAE g $\left.{ }^{-1}\right)$. Este resultado é similar aos valores encontrados para os méis in natura da abelha sem ferrão Melipona subnitida. ${ }^{8}$ $\mathrm{O}$ teor de flavonoides variou de 0,15-0,18 miligramas equivalente a quercetina por cada grama de mel ( $\mathrm{mg} \mathrm{EQ} \mathrm{g}^{-1}$ de mel).

As atividades antirradicalares das amostras de mel estão na Tabela 6. A $\mathrm{CE}_{50}$ variou de 4,21-5,51 $\mathrm{mg} \mathrm{mL}^{-1}$ (ABTS) e 7,19-14,32 $\mathrm{mg} \mathrm{mL}^{-1}$ (DPPH). Para os resultados obtidos com o radical DPPH os valores da $\mathrm{EC}_{50}$ também estão próximos dos valores encontrados para os méis da Melipona subnitida. ${ }^{8}$ Provavelmente a atividade antirradicalar está relacionada com os flavonoides presentes nos méis, uma vez que foram identificados vários, dentre eles a quercetina e luteolina, conhecidos por apresentar estas atividades. ${ }^{37}$ Todos os resultados obtidos com os méis de abelha branca são inéditos. Sobre a realização dos testes teor de fenólicos totais, teor de flavonóides e avaliação das atividades antirradicalares nos méis de abelha branca in natura, na literatura só existe relato sobre esta metodologia para os méis da abelha sem ferrão jandaíra. ${ }^{8}$ As atividades antirradicalares apresentadas pelas cinco amostras de méis indicam 

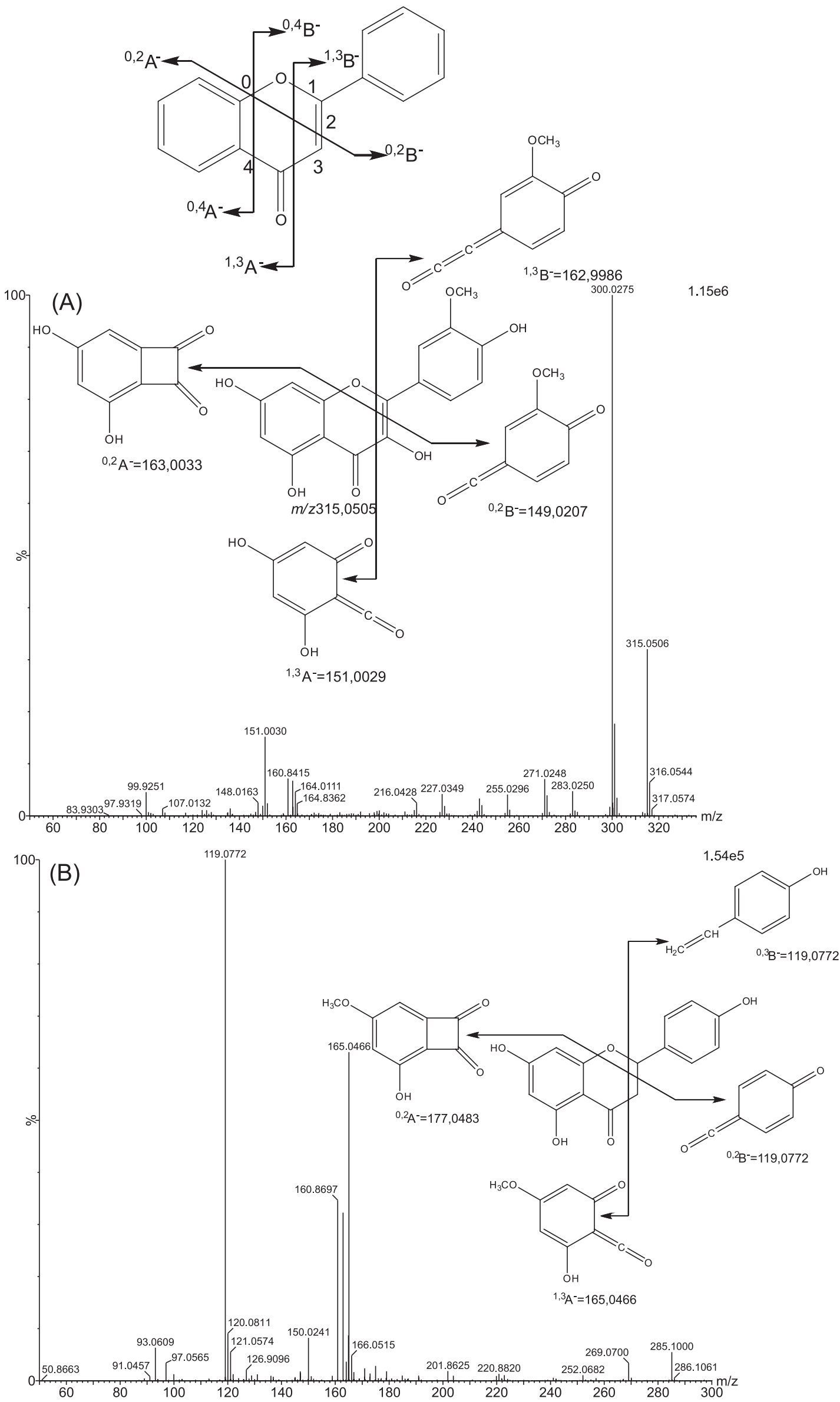

Figura 3. Espectro de íons produtos de isoramnetina (A) e 7-metoxi-naringenina (B) 


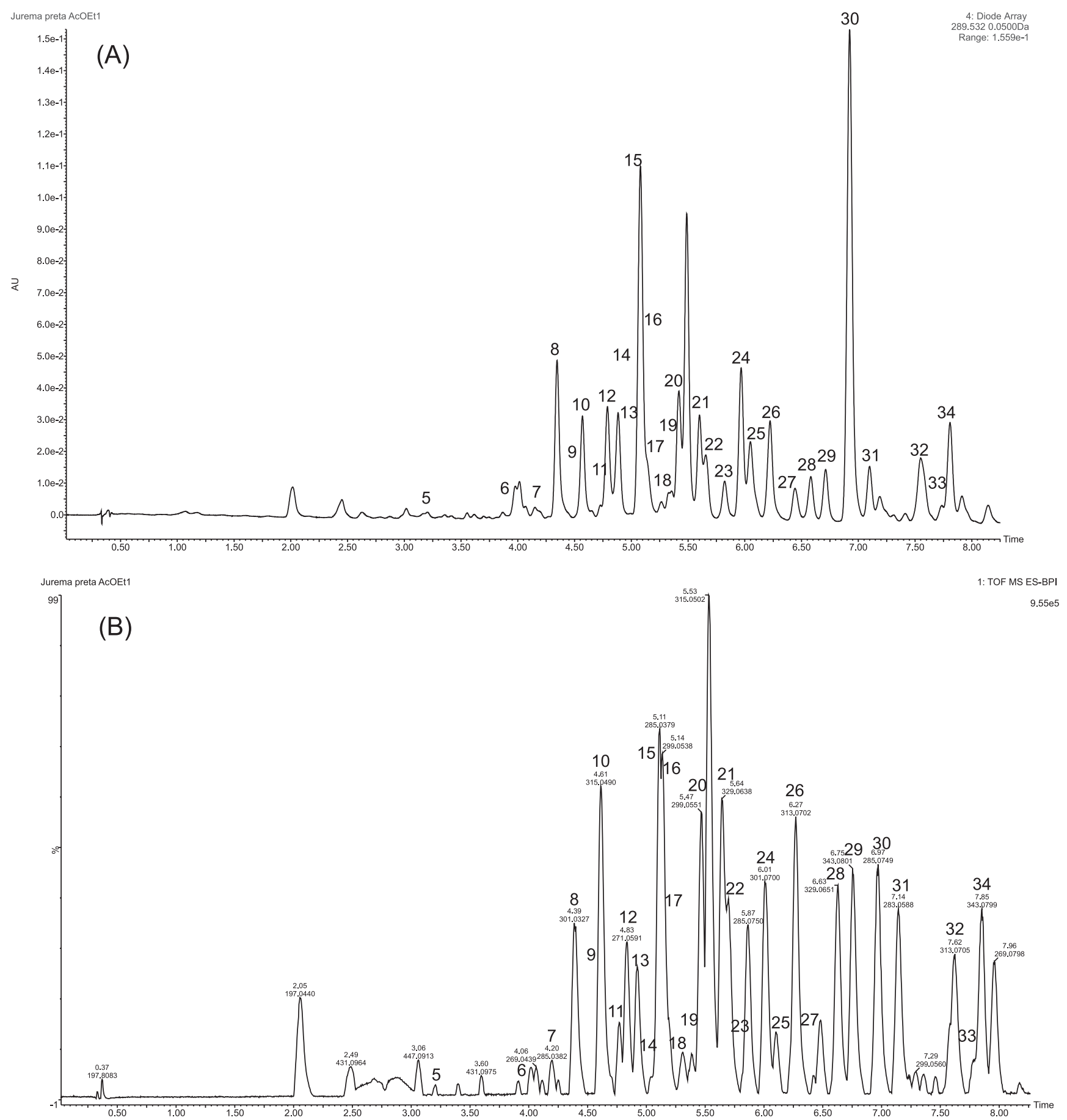

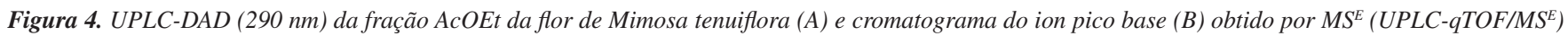
em modo negativo

Tabela 6. Teor de flavonoides, fenólicos totais e atividade antirradicalar (DPPH e ABTS) dos méis da abelha branca

\begin{tabular}{lcccc}
\hline Amostras & Teor de fenólicos $\left(\mathrm{mg} \mathrm{EAG} \mathrm{g}{ }^{-1} \mathrm{Mel}\right)$ & Teor de flavonoides $\left(\mathrm{mg} \mathrm{EQ} \mathrm{g}{ }^{-1} \mathrm{Mel}\right)$ & $\mathrm{DPPH} \mathrm{CE}_{50}\left(\mathrm{mg} \mathrm{mL}^{-1}\right)$ & $\mathrm{ABTS} \mathrm{CE}_{50}\left(\mathrm{mg} \mathrm{mL}^{-1}\right)$ \\
\hline B1 & $1,21 \pm 0,02$ & $0,15 \pm 0,02$ & $14,32 \pm 0,82$ & $4,76 \pm 0,04$ \\
B2 & $1,16 \pm 0,01$ & $0,18 \pm 0,02$ & $13,81 \pm 0,31$ & $5,51 \pm 0,08$ \\
B3 & $1,21 \pm 0,06$ & $0,16 \pm 0,01$ & $13,17 \pm 0,81$ & $4,21 \pm 0,97$ \\
B4 & $1,15 \pm 0,03$ & $0,16 \pm 0,00$ & $10,38 \pm 0,45$ & $5,26 \pm 0,19$ \\
B5 & $1,23 \pm 0,02$ & $0,15 \pm 0,00$ & $15,24 \pm 0,11$ & $4,95 \pm 0,08$ \\
\hline
\end{tabular}

${ }^{a}$ valor médio \pm desvio padrão, $\mathrm{n}=3$. 
o potencial antioxidante do mel de abelha branca com tipo polínico jurema preta predominante.

\section{CONCLUSÃO}

Os méis da abelha branca mostraram a predominância de dois principais tipos polínicos, um relativo a plantas do gênero Schinus (Anacardeaceae), com variação de 11 a $36 \%$, e outro de Mimosa tenuiflora (Fabaceae), com variação de 29 a 34\%. As análises físico-químicas mostraram um perfil similar nas amostras analisadas. Entre os minerais analisados, o potássio foi o mais abundante, seguido do magnésio. Os méis apresentam potencial antioxidante, demonstrado pela atividade antirradicalar frente ao DPPH e ABTS. Pela análise de UPLC-DAD-qTOF-MS/MS foi possível identificar 32 principais substâncias, sendo todos derivados fenólicos. Apenas três compostos não são flavonoides agliconas: ácido elágico, dihidroxicumarina e isoramnetina-glicosídeo. Dos 29 flavonóides agliconas identificados, 21 são flavonas/flavonois e 8 são flavanonas/flavanonois. Os principais flavonóides presentes no mel de abelha branca são também os constituintes principais das flores da jurema preta, indicando o forrageamento específico das abelhas nativas por espécies vegetais nativas da caatinga brasileira.

\section{MATERIAL SUPLEMENTAR}

Cromatogramas e espectros de massas MS/MS das análises (Figuras 1S e 2S) estão disponíveis em http://www.quimicanova.sbq. org.br, em formato PDF, com acesso livre.

\section{AGRADECIMENTOS}

Ao Conselho Nacional de Desenvolvimento Científico e Tecnológico (CNPq/MDA/SPM-PR número 462941/2014-0), projeto "MeliApis: inserção e capacitação da mulher em atividade da agricultura familiar em municípios do território do sertão próximo ao Rio São Francisco", à Fundação de Amparo à Ciência e Tecnologia do Estado de Pernambuco (FACEPE-PRONEM número 0741.1.06/14), Centro de Apoio à Pesquisa-Universidade Federal Rural de Pernambuco (CENAPESQ-UFRPE) e Coordenação de Aperfeiçoamento de Pessoal de Nível Superior (CAPES).

\section{REFERÊNCIAS}

1. Silveira, F. A.; Melo, G. A. R.; Almeida, E. A. B.; Abelhas brasileiras: sistemática e identificação; Silveira, F. A., org.; $1^{\mathrm{a}}$ ed., Belo Horizonte, 2002.

2. Zanella, F. C. V.; Martins, C. F. Em Ecologia e Conservação da Caatinga; Leal, I. R., Tabarelli, M., Silva, J. M. C., eds.; Editora Universitária (Universidade Federal de Pernambuco): Recife, 2003.

3. Maia-Silva, C.; Silva, C. I.; Hrncir, M.; Queiroz, R. T.; ImperatrizFonseca, V. L; Guia de plantas visitadas por abelhas na caatinga; Carbogim, J. B. P., org.; 1ª ed; Fundação Brasil Cidadão: Fortaleza, 2012.

4. Oliveira, F.; Tese de Doutorado, Universidade Federal do Paraná, Brasil, 2003.

5. Camargo, R. C. R.; Oliveira, K. L.; Berto, M. I.; Brazilian J. Food Technol. 2017, 20, e201615.

6. https://www.defesa.agricultura.sp.gov.br/legislacoes/resolucao-saa-52de-3-de-outubro-de-2017,1114.html, acessada em agosto de 2019.

7. Caldas, F. R. L.; Augusto Filho, F.; Facundo, H. T.; Alves, R. F.; Santos, F. A. R.; Silva, G. R.; Camara, C. A.; Silva, T. M. S.; Quim. Nova 2019, 42,49 .
8. Silva, T. M. S.; dos Santos, F. P.; Evangelista-Rodrigues, A.; Silva, E. M. S.; Silva, G. S.; de Novais, J. S.; Santos, F. A. R.; Camara, C. A.; J. Food Compos. Anal. 2013, 29, 10.

9. da Silva, I. A. A.; Silva, T. M. S.; Camara, C. A.; Queiroz, N.; Magnani, M.; de Novais, J. S.; Soledade, L. E. B.; Lima, E. O.; de Souza, A. L.; de Souza, A. G.; Food Chem. 2013, 141, 3552.

10. Souza, S. A.; Camara, C. A.; Silva, E. M. S.; Silva, T. M. S.; EvidenceBased Complement. Altern. Med. 2013, 2013, 1.

11. Souza, S. A.; Silva, T. M. G.; Silva, E. M. S.; Camara, C. A.; Silva, T. M. S.; Phytochem. Anal. 2018, 29, 549.

12. Souza, S. A.; Dias, T. L. M. F.; Silva, T. M. G.; Falcão, R. A.; AlexandreMoreira, M. S.; Silva, E. M. S.; Camara, C. A.; Silva, T. M. S.; Sociobiology 2014, 61, 560.

13. da Silva, G. R.; da Natividade, T. B.; Camara, C. A.; Silva, E. M. S.; Santos, F. A. R.; Silva, T. M. S.; Food Nutr. Sci. 2014, 5, 1015.

14. Zenebon, O.; Pascuet, N. S.; Tiglea, P.; Métodos Físico-Químicos para Análise de Alimentos, $4^{\mathrm{a}}$ ed., $1^{\mathrm{a}}$ Ed. Digital, Instituto Adolfo Lutz: São Paulo, 2008.

15. Erdtman, G.; Sven. Bot. Tidskr. 1960, 54, 561.

16. Silva, T. M. G.; Silva, P. R.; Camara, C. A.; Silva, G. S.; Santos, F. A. R.; Silva, T. M. S.; Rev. Virtual Quim. 2014, 6, 1370.

17. Vermerris, W.; Nicholson, R. Em Phenolic Compound Biochemistry; Springer: New York, 2006; p. 151-196.

18. Barth, O. M.; Sci. Agric. 2004, 61, 342.

19. Teixeira, A. F. R.; Oliveira, F. F.; Viana, B. F.; Neotrop. Entomol. 2007, 36,675 .

20. Crane, E.; O livro do mel, $2^{\mathrm{a}}$ ed., Livraria Nobel: São Paulo, 1983.

21. Khalil, M. I.; Moniruzzaman, M.; Boukraâ, L.; Benhanifia, M.; Islam, M. A.; Islam, M. N.; Sulaiman, S. A.; Gan, S. H.; Molecules 2012, 17, 11199 .

22. Lira, A. F.; Sousa, J. P. L. M.; Lorenzon, M. C. A.; Vianna, C. A. F. J.; Castro, R. N.; Acta Vet. Bras. 2014, 8, 169

23. Franco, B. D. G. M.; Landgraf, M.; Microbiologia dos alimentos; Atheneu: São Paulo, 2005.

24. Acquarone, C.; Buera, P.; Elizalde, B.; Food Chem. 2007, 101, 695.

25. Yadata, D.; Food Sci. Technol. 2014, 2, 59.

26. Holanda, C. A.; Oliveira, R. A.; Costa, M. C. P.; Quim. Nova 2012, 35, 55.

27. Felsner, M. L.; Cano, C. B.; Bruns, R. E.; Watanabe, H. M.; AlmeidaMuradian, L. B.; Matos, J. R.; J. Food Compos. Anal. 2004, 17, 737.

28. Silva, A. S.; Alves, C. N.; Fernandes, K. G.; Müller, R. C. S.; J. Braz. Chem. Soc. 2013, 24, 1135.

29. Liberato, M. C. T. C.; Morais, S. M.; Magalhães, C. E. C.; Magalhães, I. L.; Cavalcanti, D. B.; Silva, M. M. O.; Food Sci. Technol. 2013, 33, 38.

30. Nanda, V.; Sarkar, B. C.; Sharma, H. K.; Bawa, A. S.; J. Food Compos. Anal. 2003, 16, 613.

31. Brasil, Resolução RDC n 54, de 12 de novembro de 2012, ANVISA, Diário Oficial da União, 219, Brasília, 13 novembro de 2012, Seção 1, p.122.

32. Wyrepkowski, C. C.; da Costa, D. L. M. G.; Sinhorin, A. P.; Vilegas, W.; De Grandis, R. A.; Resende, F. A.; Varanda, E. A.; dos Santos, L. C.; Molecules 2014, 19, 16039.

33. Xu, T.; Yang, M.; Li, Y.; Chen, X.; Wang, Q.; Deng, W.; Pang, X.; Yu, K.; Jiang, B.; Guan, S.; Guo, D. A.; Rapid Commun. Mass Spectrom. 2013, 27, 2297.

34. Tsimogiannis, D.; Samiotaki, M.; Panayotou, G.; Oreopoulou, V.; Molecules 2007, 12, 593.

35. Ma, Y. L.; Li, Q. M.; Van Den Heuvel, H.; Claeys, M.; Rapid Commun. Mass Spectrom. 1997, 11, 1357.

36. Huck, C. W.; Huber, C. G.; Ongania, K. H.; Bonn, G. K.; J. Chromatogr. A 2000, 870, 453.

37. Burda, S.; Oleszek, W.; J. Agric. Food Chem. 2001, 49, 2774. 